(C) 2018 by the Arizona Board of Regents on behalf of the University of Arizona. This is an Open Access article, distributed under the terms of the Creative Commons Attribution licence (http://creativecommons. org/licenses/by/4.0/), which permits unrestricted re-use, distribution, and reproduction in any medium, provided the original work is properly cited.

\title{
ATTENDEES OF FINAL CAST SYMPOSIUM
}

\begin{tabular}{|c|c|}
\hline Name & Organization \\
\hline Ambard, Antoine & $\mathrm{EdF}$ \\
\hline Andrey Bukaemskiy & Forschungszentrum Juelich GmbH (FZJ) \\
\hline Avramova, Penka & State Enterprise Radioactive Waste \\
\hline Bererd, Nicolas & IPNL \\
\hline Buckau, Gunnar & $\mathrm{EC}$ \\
\hline Bucur, Crina & Institute for Nuclear Research (RATEN ICN) \\
\hline Caes, Sebastien & SCK.CEN \\
\hline Capouet, Manuel & Ondraf/Niras \\
\hline Charlet, Laurent & University of Grenoble \\
\hline Charmoillaux, Mathieu & $\mathrm{EdF}$ \\
\hline Chwas, Andrzej & Department of Nuclear Energy \\
\hline Clark, Ally & MCM \\
\hline Comte, Jérôme & CEA \\
\hline Cunado, Miguel & ENRESA \\
\hline Cvetković, Benjamin & PSI \\
\hline Davies, Christophe & European Commission \\
\hline de Canniere, Pierre & FANC \\
\hline de Visser-Tynova, Eva & NRG \\
\hline Demoli, Dino & Ministry of Construction and Physical Planning \\
\hline Diaconu, Daniela & Institute for Nuclear Research (RATEN ICN) \\
\hline Diomidis, Nikitas & NAGRA \\
\hline Druyts, Frank & SCK.CEN \\
\hline Feldbaumer, Eduard & ENSI \\
\hline Fugaru, Viorel & IFNN-HH \\
\hline Fulger, Manuela & Institute for Nuclear Research (RATEN ICN) \\
\hline Gaillard, Clotilde & IPNL \\
\hline Gaus, Irina & NAGRA \\
\hline Grigaliuniene, Dalia & LEI \\
\hline Hajdas, Irka & ETH Zurich \\
\hline Hart, Jaap & NRG \\
\hline Heikola, Tiina & VTT \\
\hline Herm, Michel & Karlsruhe Institute for Nuclear Waste Disposal \\
\hline Hiroyoshi, Ueda & $\begin{array}{l}\text { Radioactive Waste Management Funding and } \\
\text { Research Center (RWMC) }\end{array}$ \\
\hline Huttunen, Jani & Posiva Oy \\
\hline Källström, Klas & SKB \\
\hline Kasprzak, Laetitia & CEA \\
\hline King, Fraser & Integrity Corrosion Consulting Ltd \\
\hline Lagzdina, Elena & Center of Physical Sciences \& Technology \\
\hline Laurent, Gérard & Integrated Nuclear Engineering Solutions \\
\hline Lebeau, Diane & CEA \\
\hline Legand, Solène & CEA \\
\hline Leganés Nieto, Jose Luis & ENRESA \\
\hline Leung, Helen & NWMO \\
\hline
\end{tabular}


Levizzari, Riccardo

Magro Lozano, Enrique

Mateeva, Mayia

Mibus, Jens

Molnar, Peter

Moncoffre, Nathalie

Muňoz Serrano, José Vicente

Nakabayashi, Ryo

Narkunas, Ernestas

Necib, Sophia

Neeft, Erika

Norris, Simon

Nummi, Olli

Payne, Liam

Petit, Luarent

Pipon, Yves

Plaisir, Cyril

Plukiene, Rita

Postolache, Christian

Reiller, Pascal

Rizzo, Antonietta

Robinet, Jean-Charles

Rodríguez, Marina

Rübel, André

Sakuragi, Tomofumi

Schumacher, Stephan

Somers, Joe

Spahiu, Kastriot

Suzuki-Muresan, Tomo

Swanton, Steve

Szidat, Soenke

Toulhoat, Nelly

Večerník, Petr

Vokál, Antonín

Weetjens, Eef

Wieland, Erich

Zlobenko, Borys
ENEA

CIEMAT

BNRA

NAGRA

Public Limited Company for Radioactive Waste Management IPNL

ENRESA

Central Research Institute of Electric Power Industry

LEI

ANDRA

COVRA

RWM

Fortum

RWM

EdF

IPNL

Ariane Group

Center of Physical Sciences \& Technology

IFNN-HH

CEA

ENEA

ANDRA

CIEMAT

GRS

RWMC

ANDRA

European Commission

SKB

University of Nantes

Wood

University of Bern

IPNL

ÚJV Řež, a. s.

Súrao

SCK.CEN

PSI

IEG NASU 


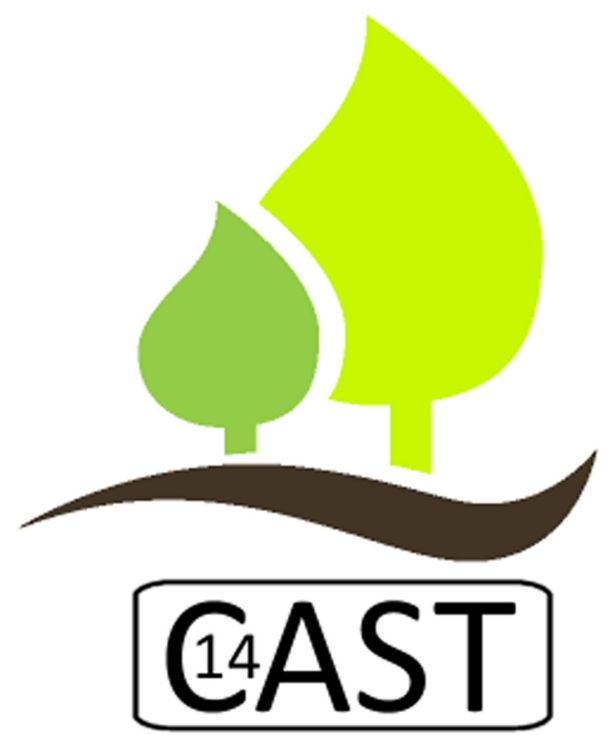

The CArbon-14 Source Term Project

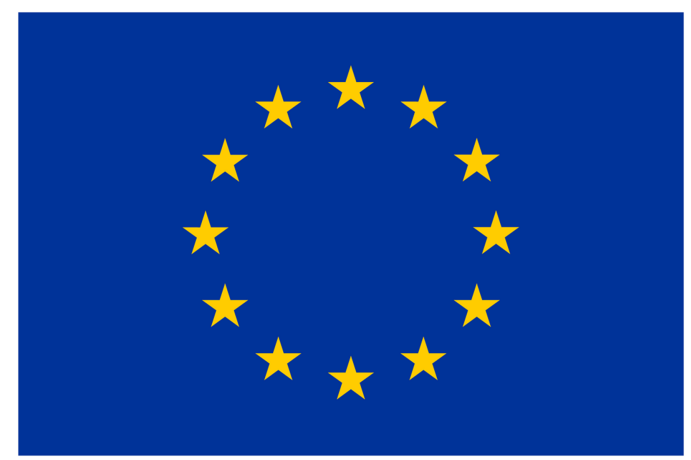

This project has received funding from the Euratom research and training programme 2007-2013 under grant agreement no. 604779, the CAST project. 


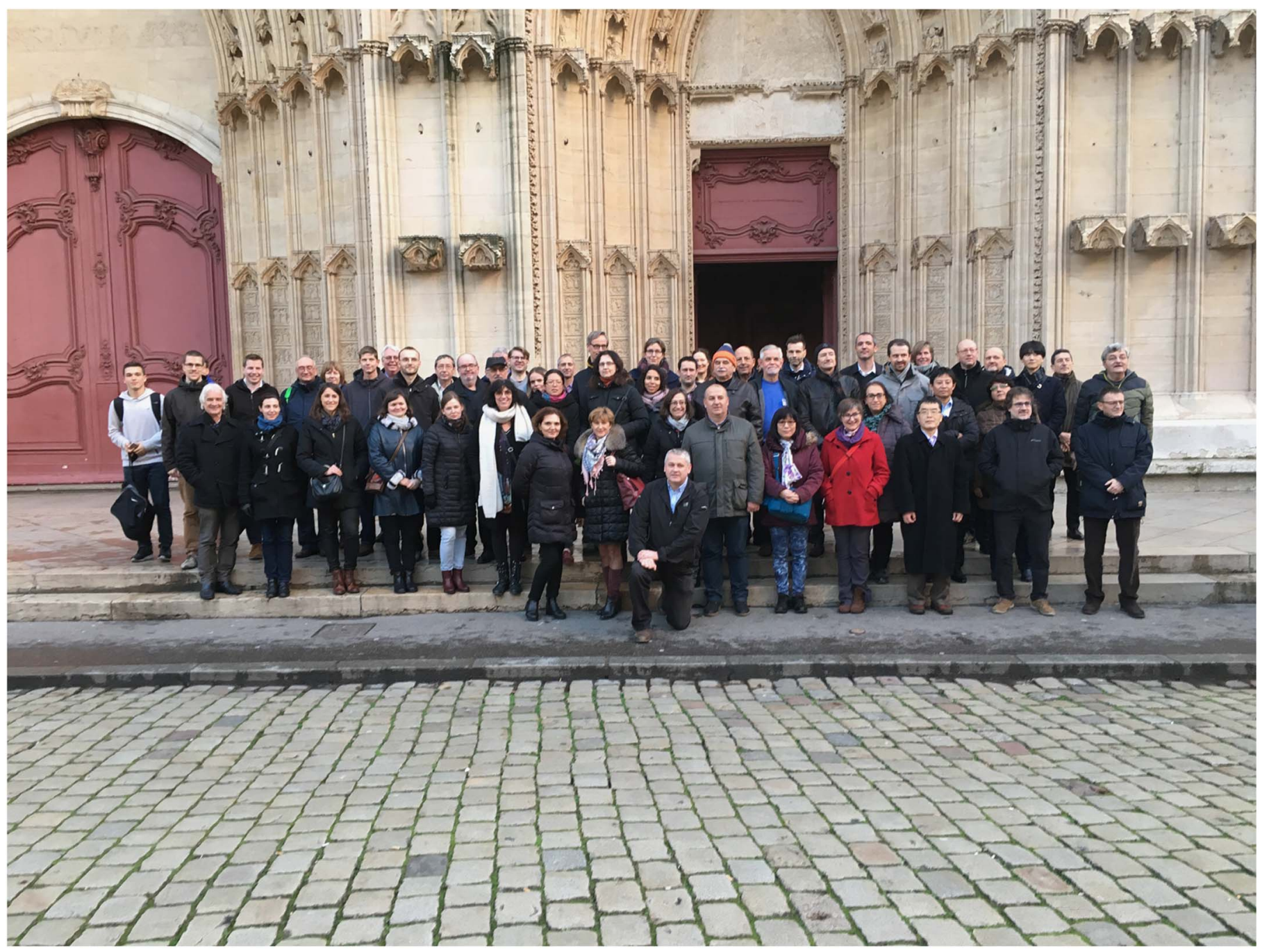

The attendees of the January 2018 CArbon-14 Source Term (CAST) Final Symposium held in Lyon, France. 